\title{
CALIBERS OF COMPACT SPACES ${ }^{1}$
}

BY

\section{S. ARGYROS AND A. TSARPALIAS}

\begin{abstract}
Let $X$ be a compact Hausdorff space and $\kappa$ its Souslin number. ${ }^{2}$ We prove that if $\alpha$ is a cardinal such that either $\alpha$ and $\operatorname{cf}(\alpha)$ are greater than $\kappa$ and strongly $\kappa$-inaccessible or else $\alpha$ is regular and greater than $\kappa$, then $X$ has $(\alpha, \sqrt[\kappa]{\alpha})$ caliber.

Restricting our interest to the category of compact spaces $X$ with $S(X)=\omega^{+}$(i.e. $X$ satisfy the countable chain condition), the above statement takes, under G.C.H., the following form.

For any compact space $X$ with $S(X)=\omega^{+}$, we have that

(a) if $\alpha$ is a cardinal and $\operatorname{cf}(\alpha)$ does not have the form $\beta^{+}$with $\operatorname{cf}(\beta)=\omega$, then $\alpha$ is caliber for the space $X$.

(b) If $\varepsilon=\beta^{+}$and $\operatorname{cf}(\beta)=\omega$ then $(\alpha, \beta)$ is caliber for $X$.

A related example shows that the result of (b) is in a sense the best possible.
\end{abstract}

Introduction. Let $X$ be a topological space and $\alpha, \beta$ cardinals. We say that the pair $(\alpha, \beta)$ is caliber for $X$ (or $X$ has $(\alpha, \beta)$ caliber) if every family $\left\{V_{\xi}: \xi<\alpha\right\}$ of nonempty open subsets of $X$ has a subfamily $\left\{V_{\xi}: \xi \in B\right\}$ such that $\bigcap_{\xi \in B} V_{\xi} \neq \varnothing$ and $|B|=\beta$.

Šanin in [6] has proved that if $\alpha$ is a regular uncountable cardinal then $(\alpha, \alpha)$ (or simply $\alpha$ ) is caliber for a product of topological spaces iff $\alpha$ is caliber for each factor. Shelah in [7] has extended this result to singular cardinals $\alpha$ in a smaller class, namely the class of powers of topological spaces.

An example of Gerlits shows that Shelah's theorem fails for arbitrary products.

In this paper we study calibers of compact spaces. The main results are the following.

THEOREM 2.5. Let $\alpha$ be a cardinal and $X$ a compact Hausdorff space with $S(X)=\kappa$. If $\alpha$ and $\operatorname{cf}(\alpha)$ are strongly $\kappa$-inaccessible and $\operatorname{cf}(\alpha)>\kappa$, then $\alpha$ is a caliber for $X$.

THEOREM 3.9. Let $\alpha, \kappa$ be cardinals with $\kappa<\alpha=\operatorname{cf}(\alpha)$ and let $X$ be a compact Hausdorff space with $S(X)=\kappa ;$ then $(\alpha, \sqrt[\kappa]{\alpha})$ is caliber for $X$.

The proofs of these theorems make use of infinitary combinatorial methods. The main combinatorial tool is Lemma 1.1 which is a consequence of ramification systems.

A consequence of Theorem 2.5 is an extension, under the G.C.H., of Shelah's result in the products of arbitrary compact Hausdorff spaces.

Received by the editors August 25, 1979 and, in revised form, October 18, 1980.

1980 Mathematics Subject Classification. Primary 54A25, 05A17, 06E10.

' The results of this paper were announced in Notices Amer. Math. Soc. 25 (1978), A-234.

${ }^{2}$ All given terms and notation are explained in $\$ \S 0$ and 3. 
Another application is the negative, in general, answer in the problem of the determination of possible calibers for the class of compact spaces. More precisely Comfort posed in [3] the problem of the determination of possible calibers on arbitrary topological spaces. Shelah in [7] and S. Broverman, J. Ginsburg, K. Kunen and F. D. Tall in [2] gave a positive answer to Comfort's problem. If we restrict this problem in the class of compact Hausdorff spaces then from our results follows that the answer is negative.

In the fourth paragraph of the paper, among others, we deal with some variations of the above problem that possibly have a positive answer. In the same section we prove that a class of topological spaces fails to have $(\alpha, \alpha)$ caliber for $\alpha=\beta^{+}$and $\operatorname{cf}(\beta)=\omega$. Hence we show that the estimation of Theorem 2.5 is in a sense the best possible.

The last section mainly contains a coherent picture of the results from a settheoretical point of view.

0. The ordinals are defined in such a way that an ordinal is the set of smaller ordinals. A cardinal is an ordinal not in one-to-one correspondence with any smaller ordinal.

We call the cofinality of a cardinal $\alpha$ and we denote by $\operatorname{cf}(\alpha)$ the least cardinal $\beta$ such that there exists a family $\left\{\alpha_{\xi}: \xi<\beta\right\}$ of cardinals less than $\alpha$ such that $\alpha=\sum_{\xi<\beta} \alpha_{\xi}$.

A cardinal $\alpha$ is regular if $\alpha=\operatorname{cf}(\alpha)$ and singular if $\operatorname{cf}(\alpha)<\alpha$. The first cardinal strictly greater than $\beta$ is denoted by $\beta^{+}$. A cardinal $\alpha$ is a successor cardinal if it is of the form $\alpha=\beta^{+}$for some $\beta$. Every successor cardinal is regular. The cardinality of the natural numbers is denoted by $\omega$. The cardinality of a set $A$ is denoted by $|A|$. We denote by $\mathscr{P}(\alpha)\left(\operatorname{resp} . \mathscr{P}_{\kappa}(\alpha)\right)$ the set of all subsets of $\alpha$ (resp. the set of all subsets of $\alpha$ of cardinality less than $\kappa)$ and by $\mathscr{P}_{\kappa}^{*}(\alpha)$ the set $\mathscr{P}_{\kappa}(\alpha) \backslash\{\varnothing\}$.

The cardinality of $\mathscr{P}(\alpha)$ is denoted by $2^{\alpha}$; more generally the cardinality of the set of all functions from (a set of cardinality) $\alpha$ to (a set of cardinality) $\alpha$ is denoted by $\beta^{\alpha}$.

We set $\alpha^{\underline{\kappa}}=\sum\left\{\alpha^{\lambda}: \lambda<\kappa\right\}$. It is known that $\left(\alpha^{\underline{\kappa}}\right)^{\underline{\kappa}}=\alpha^{\underline{\kappa}}$ for regular cardinals $k$. The continuum hypothesis is the statement $\omega^{+}=2^{\omega}$. The generalized continuum hypothesis is the statement $\alpha^{+}=2^{\alpha}$ for all infinite cardinals $\alpha$.

A cardinal $\alpha$ is strong limit if for every $\beta<\alpha$ : $2^{\beta}<\alpha$.

If $\alpha$ and $\kappa$ are cardinals then $\alpha$ is called strongly $\kappa$-inaccessible if $\beta^{\lambda}<\alpha$ for any $\beta<\alpha$ and $\lambda<\kappa$. If in addition $\alpha>\kappa$ then we write $\alpha \gg \kappa$ (or $\kappa \ll \alpha$ ). Thus e.g. if $\alpha=\left(2^{k}\right)^{+}$then $\alpha \gg \kappa^{+}$; and every infinite cardinal is strongly $\omega$-inaccessible.

The weight of a topological space $X$, denoted by $w(X)$, is the least cardinal $\alpha$ such that there is a base of the topology of $X$ with cardinality $\alpha$ and the density character of $X$, denoted by $d(X)$, is the least cardinal $\beta$ with the property that there is a dense subset of $X$ with cardinality $\beta$. The Souslin number of a topological space $X$, denoted by $S(X)$, is the least cardinal $\kappa$ such that there is no family of cardinality $\kappa$ of pairwise disjoint nonempty open subsets of $X$.

The theorem of Erdös and Tarski states that the Souslin number of an infinite Hausdorff topological space is a regular uncountable cardinal. A space $X$ satisfies the countable chain condition (c.c.c.) if $S(X) \leqslant \omega^{+}$. 
Let $X$ be a topological space and $\alpha, \beta$ cardinals; we say that $X$ has precaliber $(\alpha, \beta)$ (caliber $(\alpha, \beta))$ if, for every family $\left\{V_{\xi}: \xi<\alpha\right\}$ of nonempty open subsets of $X$, there is $B \subset \alpha$ with $|B|=\beta$ such that the family $\left\{U_{\xi}: \xi \in B\right\}$ has the finite intersection property (has nonempty intersection resp.). It is clear that a compact Hausdorff space has precaliber $(\alpha, \beta)$ if and only if it has caliber $(\alpha, \beta)$. We say that a space $X$ has precaliber $\alpha$ (caliber $\alpha$ resp.) if $X$ has precaliber $(\alpha, \alpha)$ (caliber $(\alpha, \alpha)$ resp.).

1.

1.1. Lemma. Let $\alpha, \rho$ be infinite regular cardinals and $\kappa$ be a cardinal with $\kappa \ll \rho \leqslant \alpha$. We also assume that for every $A \subset \alpha$ with $|A|=\alpha$ there is a partition $\mathscr{P}_{A}$ of $A$, such that $\left|\mathscr{P}_{A}\right|<\rho$. Then there is a family $\left\{A_{\eta}: \eta<\kappa\right\}$ of subsets of $\alpha$ such that

$$
\begin{gathered}
\left|A_{\eta}\right|=\alpha \quad \text { for all } \eta<\kappa, \quad A_{\eta+1} \in \mathscr{P}_{A_{\eta}} \text { for } \eta<\kappa, \\
A_{\eta^{\prime}} \subset A_{\eta} \text { for } \eta<\eta^{\prime}<\kappa .
\end{gathered}
$$

Proof. For every $A \subset \alpha$ with $|A|<\alpha$, we denote by $\mathscr{P}_{A}$ the $\{A\}$ and so we extend the notation $\mathscr{P}_{A}$ to every set $A$ subset of $\alpha$.

Inductively, for every $\eta<\kappa$, we define a set $Q_{\eta} \subset \mathcal{P}(\alpha)$ as follows.

We set $\mathbb{Q}_{0}=\{\alpha\}$, if $\eta<\kappa$ and $\mathbb{Q}_{\eta}$ has been defined, then we set

$$
\mathbb{Q}_{\eta+1}=\cup\left\{\mathscr{P}_{A}: A \in \mathbb{Q}_{\eta}\right\} .
$$

If $\eta<\kappa$ is a limit ordinal and for every $\xi<\eta \mathscr{Q}_{\xi}$ has been defined put

$$
\mathbb{Q}_{\eta}=\left\{\bigcap_{\xi<\eta} A_{\xi}: A_{\xi} \in \mathbb{Q}_{\xi} \text { for } \xi<\eta\right\} .
$$

We claim that for every $\eta<\kappa,\left|\mathbb{Q}_{\eta}\right|<\rho$.

In fact, indeed if $\eta<\kappa$ and if we assume that $\left|\mathbb{Q}_{\eta}\right|<\rho$ then one easily verifies that also $\left|\mathbb{Q}_{\eta+1}\right|<\rho$.

Now, let us assume that $\eta$ is a limit ordinal and for every $\xi<\eta$ we have that $\left|\mathbb{Q}_{\xi}\right|<\rho$. Then we set $\beta=\sup \left\{\left|\mathbb{Q}_{\xi}\right|: \xi<\eta\right\}$ and we notice that $\beta<\rho$ since $\rho$ is a regular cardinal.

We also define a map

$$
\phi_{\eta}: \mathbb{Q}_{\eta} \rightarrow \prod_{\xi<\eta} \mathbb{Q}_{\xi}
$$

with the rule

$$
\phi_{\eta}\left(\bigcap_{\xi<\eta} A_{\xi}\right)=\left\{A_{\xi}: \xi<\eta\right\} .
$$

Obviously $\phi_{\eta}$ is one-to-one and since $\rho$ is strongly $\kappa$-inaccessible we have that

$$
\left|\mathbb{Q}_{\eta}\right| \leqslant\left|\prod_{\xi<\eta} \mathbb{Q}_{\xi}\right|=\prod_{\xi<\eta}\left|\mathscr{Q}_{\xi}\right| \leqslant \beta^{|\eta|}<\rho .
$$

This completes the proof of the claim.

Setting now $\mathbb{Q}=\cup_{\eta<\kappa} \mathbb{Q}_{\eta}$ we have that $|\mathfrak{Q}|<\rho$. Consequently the set $\mathbb{Q}^{\prime}=$ $\{A \in \mathbb{Q}:|A|<\alpha\}$ also has cardinality less than $\rho$ and since $\alpha$ is a regular cardinal it follows that $\left|\cup \mathbb{Q}^{\prime}\right|<\alpha$. We choose an $x \in \alpha \backslash \cup \mathbb{Q}^{\prime}$. For every $\eta<\kappa$ there is a 
unique $A_{\eta}(x)$ element of $\mathbb{Q}_{\eta}$ such that $x \in A_{\eta}(x)$. It is easily seen that the family $\left\{A_{\eta}(x): \eta<\kappa\right\}$ has the desired properties.

1.1.a. LEMMA. The conclusion of Lemma 1.1 remains true in the case $\alpha=\lambda=\kappa$ and $\kappa$ is a weakly compact cardinal.

Indeed, in the construction of the sets $\left\{Q_{\sigma}: \sigma<\kappa\right\}$ we only need that for $\lambda, \mu$ cardinals less than $\kappa$ it holds $\lambda^{\mu}<\kappa$ while the existence of family $\left\{A_{\sigma}: \sigma<\kappa\right\}$ that satisfies the conclusion of the lemma follows from properties of weakly compact cardinals.

1.2. Lemma. Let $\alpha$ be an (infinite) singular cardinal, $\beta<\alpha$ and $\kappa \ll \alpha$. If $\kappa=\omega$ or $\kappa<\operatorname{cf}(\alpha)$ then there is a regular cardinal $\gamma$ such that $\beta<\gamma<\alpha$ and $\kappa \ll \gamma$.

Proof. If $\delta<\alpha$ and $\lambda<\kappa$ then $\delta^{\lambda}<\alpha$; it follows that

$$
\delta^{\kappa}=\sum_{\lambda<\kappa} \delta^{\lambda}<\alpha
$$

Now set $\gamma=\left(\left(\beta^{\kappa}\right)^{\kappa}\right)^{+}$. It follows from the fact that $\alpha$ is not a successor cardinal that $\gamma<\alpha$. Since $\gamma$ is regular, to complete the proof it is enough to show that $\kappa \ll \gamma$. For $\delta<\gamma$ and $\lambda<\kappa$ we have

$$
\delta^{\lambda} \leqslant\left(\left(\beta^{\underline{\kappa}}\right)^{\underline{\kappa}}\right)^{\lambda} \leqslant\left(\left(\beta^{\underline{\kappa}}\right)^{\underline{\kappa}}\right)^{\underline{\kappa}}=\left(\beta^{\underline{\kappa}}\right)^{\underline{\kappa}}<\gamma
$$

(from the general set-theoretic relation $\left.\left(\left(\beta^{\underline{\kappa}}\right)^{\underline{\kappa}}\right)^{\underline{\kappa}}=\left(\beta^{\underline{\kappa}}\right)^{\underline{\kappa}}\right)$, as required.

1.3. Corollary. Let $\alpha$ be an (infinite) singular cardinal and $\kappa \ll \alpha$. If $\kappa=\omega$ or $\kappa<\operatorname{cf}(\alpha)$ then there is a set $\left\{\alpha_{\sigma}: \sigma<\operatorname{cf}(\alpha)\right\}$ such that

(i) $\alpha_{\sigma}$ is a regular cardinal for $\sigma<\operatorname{cf}(\alpha)$,

(ii) $\kappa \ll \alpha_{\sigma}$ for $\sigma<\operatorname{cf}(\alpha)$,

(iii) $\operatorname{cf}(\alpha)<\alpha_{\sigma^{\prime}}<\alpha_{\sigma}<\alpha$ for $\sigma^{\prime}<\sigma<\operatorname{cf}(\alpha)$, and

(iv) $\alpha=\sum_{\sigma<\mathrm{cf}(\alpha)} \alpha_{\sigma}$.

Proof. Since $\alpha$ is singular there is a family $\left\{\gamma_{\sigma}: \sigma<\operatorname{cf}(\alpha)\right\}$ of cardinals with properties (analogous to) (iii) and (iv). We set $\beta_{0}=\gamma_{0}$ and recursively, if $\sigma<\operatorname{cf}(\alpha)$ and $\alpha_{\sigma^{\prime}}$ has been defined for all $\sigma^{\prime}<\sigma$, we set

$$
\beta_{\sigma}=\max \left\{\left(\sup \left\{\alpha_{\sigma^{\prime}}: \sigma^{\prime}<\sigma\right\}\right)^{+} \gamma_{\sigma}\right\} .
$$

By Lemma 1.2 there is $\alpha_{\sigma}$ satisfying (i) and (ii) and the condition $\beta_{\sigma}<\alpha_{\sigma}<\alpha$. It is clear that the family $\left\{\alpha_{\sigma}: \sigma<\operatorname{cf}(\alpha)\right\}$ is as required.

2.

2.1. Lemma. Let $X$ be a topological space with $S(X)=\kappa \geqslant \omega$, let $\alpha$ be a regular cardinal with $\kappa \ll \alpha$, and let $\left\{U_{\xi}: \xi<\alpha\right\}$ be a family of nonempty open subsets of $X$. Then there is $\bar{\xi}<\alpha$ such that if $\beta<\alpha$ and if $\left\{B_{\zeta}: \zeta<\beta\right\}$ is a family of nonempty open subsets $U_{\xi}$, then there is $A \subset \alpha$, with $|A|=\alpha$, such that the family $\left\{U_{\xi} \cap B_{\zeta}: \xi \in A\right\}$ has the finite intersection property for all $\zeta<\beta$.

Proof. Suppose no such $\bar{\xi}<\alpha$ exists. For $A \subset \alpha$ with $|A|=\alpha$, we choose $\xi_{A} \in A$. Then, there are a cardinal $\beta_{A}<\alpha$ and a family $\left\{B_{\zeta}^{A}: \zeta<\beta_{A}\right\}$ of nonempty open 
subsets of $U_{\xi_{A}}$ such that

If $B \subset A$, and $\left\{U_{\xi} \cap B_{\zeta}^{A}: \xi \in B\right\}$ has the finite intersection property for all $\zeta<\beta_{A}$, then $|B|<\alpha$.

For $A \subset \alpha$ with $|A|=\alpha$ we set

$\mathscr{B}_{A}=\left\{\left\{B \subset A: U_{\xi} \cap B_{\zeta}^{A}: \xi \in B\right\}\right.$ has the finite intersection

property for all $\left.\xi<\beta_{A}\right\}$.

The family $\mathscr{B}_{A}$ partially ordered by set inclusion is inductive, hence there is a maximal element $B_{A} \in \mathscr{B}_{A}$. Also it is clear that $B_{A} \neq \varnothing$ and that $\left|B_{A}\right|<\alpha$.

We define

$$
\phi_{A}: A \backslash B_{A} \rightarrow \mathscr{P}_{\omega}^{*}\left(B_{A}\right) \times \beta_{A}
$$

as follows. Let $\eta \in A \backslash B_{A}$. The maximality of $B_{A}$ implies that there is a nonempty finite subset $F_{\eta}$ of $B_{A}$ and $\zeta_{\eta}<\beta_{A}$ such that

$$
\left(\bigcap_{\xi \in F_{\eta}} U_{\xi}\right) \cap B_{\xi_{\eta}}^{A} \cap U_{\eta}=\varnothing
$$

we set $\phi_{A}(\eta)=\left(F_{\eta}, \zeta_{\eta}\right)$ for $\eta \in A \backslash B_{A}$. We set

$$
\mathscr{P}_{A}=\left\{B_{A}\right\} \cup\left\{\phi_{A}^{-1}(\{(F, \zeta)\}):(F, \zeta) \in \mathcal{P}_{\omega}^{*}\left(B_{A}\right) \times \beta_{A}\right\}
$$

and we note that

$$
\mathscr{P}_{A} \text { is a partition of } A \text {, with }\left|\mathscr{P}_{A}\right|<\alpha .
$$

Since $\alpha$ is regular and $\omega \leqslant \kappa \ll \alpha$, it follows from Lemma 1.1 that there is a family $\left\{A_{i}: i<\kappa\right\}$ such that $\left|A_{i}\right|=\alpha$ for $i<\kappa, A_{i^{\prime}} \subset A_{i}$ for $i<i^{\prime}<\kappa$, and $A_{i+1} \in \mathscr{O P}_{A_{i}}$ for $i<\kappa$.

Thus, since $\left|A_{i}\right|=\alpha$ for all $i<\kappa$, we have

$$
A_{i+1}=\phi_{A_{i}}^{-1}\left(\left\{\left(F_{i}, J_{i}\right)\right\}\right)
$$

for some $\left(F_{i}, J_{i}\right) \in \mathcal{P}_{\omega}^{*}\left(B_{A_{i}}\right) \times \beta_{A_{i}}, i<\kappa$. We set

$$
V_{i}=\left(\bigcap_{\xi \in F_{i}} U_{\xi}\right) \cap B_{j_{i}} \text { for } i<\kappa,
$$

and we claim that the family $\left\{V_{i}: i<\kappa\right\}$ consists of pairwise nonempty disjoint subsets of $X$. In fact observing that for $j<\kappa V_{j} \subset U_{\xi_{A_{j}}}$ and that

$$
\xi_{A_{j}} \in A_{i+1}=\phi_{A_{i}}^{-1}\left(\left\{F_{i}, J_{i}\right\}\right)
$$

we have that

$$
V_{i} \cap U_{\xi_{A j}}=\varnothing, \text { and from this } V_{i} \cap V_{j}=\varnothing .
$$

So the claim is true and this contradicts the assumption $S(X)=\kappa$. The proof is now complete.

2.2. Remark. The conclusion of Lemma 2.1 remains true assuming that $\alpha=\kappa$ and $\alpha$ is a weakly compact cardinal. In fact applying the same methods as above and using Lemma 1.1. $\alpha$ instead of Lemma 1.1 we get the desired result. 
2.3. Corollary. Let $X$ be a topological space with $S(X)=\kappa \geqslant \omega$, and let $\alpha$ be a cardinal such that either $\alpha$ is regular with $\alpha \gg \kappa$ or else $\alpha$ is weakly compact and $\alpha \geqslant \kappa$. Then $\alpha$ is a precaliber for the space $X$.

Proof. Let $\left\{U_{\xi}: \xi<\alpha\right\}$ be a family of nonempty open subsets of $X$. Choose $\bar{\xi}<\alpha$ as in Lemma 2.1 or Remark 2.2. For $\beta=1, B_{0}=U_{\xi}$ we find an $A \subset \alpha$ with $|A|=\alpha$ such that the family

$\left\{U_{\xi} \cap U_{\xi}: \xi \in A\right\}$ has the finite intersection property. Thus $X$ has precaliber $\alpha$.

2.4. Lemma. Let $X$ be a space with $S(X)=\kappa$ and let $\alpha$ be a singular cardinal such that $X$ has precaliber $\mathrm{cf}(\alpha)$, and

there is a family $\left\{\alpha_{\sigma}: \sigma<\operatorname{cf}(\alpha)\right\}$ of cardinals such that $\alpha_{\sigma}$ is regular, $\kappa \ll \alpha_{\sigma}$ for $\sigma<\operatorname{cf}(\alpha), \alpha_{\sigma}<\alpha_{\tau}$ for $\sigma<\tau<\operatorname{cf}(\alpha)$, and $\Sigma_{\sigma<\operatorname{cf}(\alpha)} \alpha_{\sigma}=\alpha$.

Then $X$ has precaliber $\alpha$.

Proof. Let $\left\{U_{\xi}: \xi<\alpha\right\}$ be a family of nonempty open subsets of $X$. Since $\kappa \ll \alpha_{\sigma}$ and $\alpha_{\sigma}$ is regular, it follows from Lemma 2.1 that there is $J_{\sigma}<\alpha_{\sigma}$ such that

(*) if $\beta<\alpha_{\sigma}$ and if $\left\{B_{\eta}: \eta<\beta\right\}$ is a family of nonempty open subsets of $U_{J_{o}}$, then there is $A \subset \alpha_{\sigma}$ with $|A|=\alpha_{\sigma}$ such that, for each $\eta<\beta$, the family $\left\{U_{\xi} \cap B_{\eta}: \xi \in A\right\}$ has the finite intersection property.

It follows from $(*)$ that

(**) If $9 B$ is a family of open subsets of $U_{J_{\sigma}}$ having the finite intersection property, and if $|\Re|<\alpha_{\sigma}$, then there is $A \subset \alpha_{\sigma}$ with $|A|=\alpha_{\sigma}$ such that the family $\mathscr{G} \cup\left\{U_{\xi}: \xi\right.$ $\in A\}$ has the finite intersection property.

In particular,

(***) If $J_{\sigma} \in B \subset \alpha_{\sigma},|B|<\alpha_{\sigma}$, and $\left\{U_{\xi}: \xi \in B\right\}$ has the finite intersection property, then there is $A \subset \alpha_{\sigma}$ with $\left|A_{\sigma}\right|=\alpha_{\sigma}$ such that the family $\left\{U_{\xi}: \xi \in B \cup A\right\}$ has the finite intersection property.

Since $\operatorname{cf}(\alpha)$ is a precaliber for $X$, there is $S \subset \operatorname{cf}(\alpha)$ with $|S|=\operatorname{cf}(\alpha)$ such that the family $\left\{U_{J_{\sigma}}: \sigma \in S\right\}$ has the finite intersection property. Let $Z=\left\{J_{\sigma}: \sigma \in S\right\}$. Let $B$ be maximal such that $Z \subset B \subset \alpha$ and $\left\{U_{\xi}: \xi \in B\right\}$ has the finite intersection property. We claim that $|B|=\alpha$. Assume the contrary; $|B|<\alpha_{\sigma}$ for some $\sigma \in S$. By (***) there is $A \subset \alpha_{\sigma}$ with $|A|=\alpha_{\sigma}$ such that $\left\{U_{\xi}: \xi \in B \cup A\right\}$ has the finite intersection property, contradicting the maximality of $B$.

2.5. THEOREM. Let $\omega \leqslant \kappa \ll \alpha$ and $\kappa \ll \operatorname{cf}(\alpha)$ and let $X$ be a topological space with $S(X)=\kappa$. Then $\alpha$ is a precaliber for $X$.

In particular, if $X$ is a compact Hausdorff space then $\alpha$ is a caliber for $X$.

Proof. Since $\operatorname{cf}(\alpha)$ is regular and $\kappa \ll \operatorname{cf}(\alpha)$, it follows from Corollary 2.3 that $X$ has precaliber $\operatorname{cf}(\alpha)$. Since $\kappa \ll \alpha$ and $\kappa<\operatorname{cf}(\alpha)$, it follows from Corollary 1.3 that there is a family $\left\{\alpha_{\sigma}: \sigma<\operatorname{cf}(\alpha)\right\}$ of cardinals satisfying the conditions of Lemma 2.4. Hence $X$ has precaliber $\alpha$. 
2.6. TheOREM. Let $X$ be a topological space with $S(X)=\kappa \geqslant \omega$ and let $\alpha$ be a cardinal such that $\beta^{\underline{\kappa}}<\alpha$ for every cardinal $\beta<\alpha$. Then $\alpha$ is a precaliber for $X$ if and only if $\operatorname{cf}(\alpha)$ is a precaliber for $X$.

In particular, if $X$ is a compact Hausdorff space then $\alpha$ is a caliber for $X$ if and only if $\operatorname{cf}(\alpha)$ is a caliber for $X$.

Proof. If $\alpha$ is a precaliber for $X$ then obviously $\operatorname{cf}(\alpha)$ also is a precaliber for $X$. Conversely if $\operatorname{cf}(\alpha)$ is a precaliber for the space $X$ and if $\operatorname{cf}(\alpha)<\alpha$ then since $\left(\beta^{\kappa}\right)^{\kappa}<\dot{\alpha}$ it follows that $\alpha=\sum_{\sigma<\operatorname{cf}(\alpha)} \alpha_{\sigma}$ where $\alpha_{\sigma}$ is regular $\alpha_{\sigma} \gg \kappa$. So the family $\left\{\alpha_{\sigma}: \sigma<\operatorname{cf}(\alpha)\right\}$ satisfies the conditions of Lemma 2.4. Hence $X$ has precaliber $\alpha$.

2.7. Corollary. Assume the Generalized Continuum Hypothesis (G.C.H.). Let X be a topological space. Then a cardinal $\alpha$ is a precaliber for $X$ if and only if $\operatorname{cf}(\alpha)$ is a precaliber for $X$.

In particular, if $X$ is a compact Hausdorff space, then $\alpha$ is a caliber for $X$ if and only if $\operatorname{cf}(\alpha)$ is a caliber for $X$.

Proof. If $\alpha=\operatorname{cf}(\alpha)$ then the result is obvious. Assuming $\operatorname{cf}(\alpha)<\alpha$ we observe that $\kappa=S(X) \leqslant \operatorname{cf}(\alpha)$ and from G.C.H. for cardinals $\beta$ with $\operatorname{cf}(\alpha)<\beta<\alpha$ we have $\beta^{\operatorname{cf}(\alpha)}=\beta^{+}<\alpha$ and hence $\beta^{\kappa}<\alpha$. The result follows now from Theorem 2.6.

2.8. Corollary. Assume the G.C.H. Let $\left\{X_{i}: i \in I\right\}$ be a family of nonempty spaces. A cardinal $\alpha$ with $\operatorname{cf}(\alpha)>\omega$ is precaliber for $X=\prod_{i \in I} X_{i}$ if and only if $\alpha$ is a precaliber for $X_{i}$ for all $i \in I$.

In particular, if $X_{i}$ is a compact Hausdorff space, then a cardinal $\alpha$ with $\operatorname{cf}(\alpha)>\omega$ is caliber for $X$ if and only if $\alpha$ is a caliber for $X_{i}$ for all $i \in I$.

Proof. Since $\alpha$ is a precaliber for each $X_{i}$ it follows that $\operatorname{cf}(\alpha)$ is a precaliber for each of them, and from [6], $\operatorname{cf}(\alpha)$ is a precaliber for the space $X$. Using Corollary 2.7 we have that $\alpha$ is a precaliber for $X$. Conversely it is clear that if $\alpha$ is a precaliber for $X$ then $\alpha$ is a precaliber for $X_{i}$ for all $i \in I$.

REMARK. In Corollaries 2.7 and 2.8 the G.C.H. can be replaced by the assumption that the Singular Cardinals Hypothesis (S.C.H. in short) holds and $2^{\operatorname{cf}(\alpha)}<\alpha$. The above condition is weaker than the corresponding conditions of Corollaries 2.7 and 2.8 when we assume that $\operatorname{cf}(\alpha)<\alpha$.

2.9. Remark. Corollaries 2.7 and 2.8 are not true for calibers of arbitrary topological spaces in case $\alpha$ is a singular cardinal as follows from the example given in [4].

2.10. Corollary. Assume the G.C.H. Let $\alpha$ be infinite cardinal and $X$ a topological space with $S(X)=\kappa \geqslant \omega$.

(a) If $\operatorname{cf}(\alpha)>\kappa$, and if either $\operatorname{cf}(\alpha)$ is a limit cardinal or else $\operatorname{cf}(\alpha)=\beta^{+}$with $\operatorname{cf}(\beta) \geqslant \kappa$, then $\alpha$ is a precaliber for $X$.

(b) If $\operatorname{cf}(\alpha)<\kappa$ then $\alpha$ is not precaliber for X. Analogous statements for calibers hold in case $X$ is a compact Hausdorff space.

REMARK. The remaining cases in the above theorem, namely either $\operatorname{cf}(\alpha)=\kappa$ or else $\operatorname{cf}(\alpha)=\beta^{+}$with $\operatorname{cf}(\beta)<\kappa$, will be investigated in the next sections. 
2.11. Corollary. Assume the Generalized Continuum Hypothesis. Let $X$ be a topological space with $S(X)=\omega_{1}$. An infinite cardinal $\alpha$ is a precaliber for $X$, unless

(a) $\operatorname{cf}(\alpha)=\omega$

in which case $\alpha$ is not a precaliber for $X$; or

(b) $\operatorname{cf}(\alpha)=\beta^{+}$, with $\operatorname{cf}(\beta)=\omega$

in which case $\alpha$ may or may not be a precaliber for $X$.

The analogous statement for calibers holds in case $X$ is a compact Hausdorff space.

Proof. This follows from the case $\kappa=\omega^{+}$of Corollary 2.10.

3.

3.1. Definition. Let $\alpha, \kappa$ be infinite cardinal numbers. We set

$$
\sqrt[\kappa]{\alpha}=\min \left\{\beta: \beta^{\kappa} \geqslant \alpha\right\}, \quad \sqrt[\kappa]{\alpha}=\min \{\sqrt[\lambda]{\alpha}: \lambda<\kappa\}
$$

It is clear that if $\lambda<\kappa$ then $2 \leqslant \sqrt[\kappa]{\alpha} \leqslant \sqrt[\lambda]{\alpha} \leqslant \alpha$; also

$$
\sqrt[\kappa]{\alpha}=\min \left\{\beta: \text { there is } \lambda<\kappa \text { with } \beta^{\lambda} \geqslant \alpha\right\} .
$$

3.2. REMARK. If $\sqrt[\kappa]{\alpha} \leqslant \beta \leqslant \alpha$ then $\sqrt[\kappa]{\alpha}=\sqrt[\kappa]{\beta}$. (Indeed it is clear that $\sqrt[\kappa]{\beta} \leqslant \sqrt[\kappa]{\alpha}$; if $\sqrt[\kappa]{\beta}<\sqrt[\kappa]{\alpha}$ then $\sqrt[\kappa]{\alpha} \leqslant(\sqrt[\kappa]{\beta})^{\kappa}<\alpha$, and hence

$$
(\sqrt[\kappa]{\alpha})^{\kappa} \leqslant(\sqrt[\kappa]{\beta})^{\kappa \cdot \kappa}=(\sqrt[\kappa]{\beta})^{\kappa}<\alpha
$$

which is impossible.)

3.3. Proposition. Let $\alpha, \kappa$ be infinite cardinals and suppose that $\sqrt[\kappa]{\alpha}<\alpha$. Then either $\sqrt[\kappa]{\alpha}=2$ or else, setting $\beta=\sqrt[\kappa]{\alpha}$, we have $\operatorname{cf}(\beta) \leqslant \kappa<\beta$.

Proof. Suppose $\sqrt[\kappa]{\alpha}>2$, and set $\beta=\sqrt[\kappa]{\alpha}$. Then $\beta>\kappa$. We prove that $\operatorname{cf}(\beta) \leqslant \kappa$. Indeed, suppose that $\operatorname{cf}(\beta)>\kappa$. Let $\gamma$ be a regular cardinal such that $\sqrt[\kappa]{\alpha}<\gamma \leqslant \alpha$; by the above remark, we have $\sqrt[\kappa]{\gamma}=\beta$. Since $\beta^{\kappa} \geqslant \gamma$, it follows that there is a one-to-one function $f: \gamma \rightarrow \beta$. Define $\phi: \gamma \rightarrow \beta$ by $\phi(\xi)=\sup \operatorname{range}(f(\xi)(\kappa))$. Since $\operatorname{cf}(\beta)>\kappa$, $\phi$ is well defined; since $\gamma$ is regular and $\beta<\gamma$, it follows that there are $\eta_{0}<\beta$ and $A \subset \gamma$, with $|A|=\gamma$, such that $\phi(\xi)<\eta_{0}$ for all $\xi \in A$; hence $f \mid A: A \rightarrow{ }^{\kappa} \eta_{0}$. Hence $\gamma=|A| \leqslant\left|\eta_{0}\right|^{\kappa}$, and $\beta>\left|\eta_{0}\right| \geqslant \sqrt[\kappa]{\gamma}$, a contradiction.

It now follows that $\operatorname{cf}(\beta) \leqslant \kappa<\beta$.

3.4. Corollary. Let $\alpha, \kappa$ be infinite cardinals and suppose that $\sqrt[\kappa]{\alpha}<\alpha$. Then either $\sqrt[\kappa]{\alpha}=2$ or else, setting $\beta=\sqrt[\kappa]{\alpha}$ we have $\operatorname{cf}(\beta)<\kappa$.

3.5. REMARK. It is clear that if $\alpha, \kappa$ are cardinals such that $\sqrt[\kappa]{\alpha} \geqslant \omega$, then $\sqrt[\kappa]{\alpha}$ is a strongly $\kappa$ inaccessible cardinal. Furthermore, if we assume that $\kappa$ is a regular cardinal and $\omega \leqslant \sqrt[\kappa]{\alpha}<\alpha$ then $\sqrt[\kappa]{\alpha} \gg \kappa$. 
3.6. Lemma. Let $X$ be a space with $S(X)=\kappa \geqslant \omega$, let $\alpha$ be a regular cardinal such that $2<\sqrt[\kappa]{\alpha}<\alpha$, and let $\left\{U_{\xi}: \xi \in \alpha\right\}$ be a family of nonempty open subsets of $X$. Then there is $A \subset \alpha$ with $|A|=\alpha$ such that: if $\Lambda \subset A,|\Lambda| \leqslant \kappa^{\kappa}$ and $\left\{U_{\xi}: \xi \in \Lambda\right\}$ has the finite intersection property, then

$$
\mid\left\{\eta \in A:\left\{U_{\xi}: \xi \in \Lambda \cup\{\eta\}\right\} \text { has the finite intersection property }\right\} \mid=\alpha .
$$

Proof. Suppose that there is no such set $A$. For $A \subset \alpha$ with $|A|=\alpha$, there are $B_{A} \subset A$ with $\left|B_{A}\right|<\alpha$ and $\Lambda_{A} \subset A$ with $\left|\Lambda_{A}\right|<\kappa^{\kappa}$ such that $\left\{U_{\xi}: \xi \in \Lambda_{A}\right\}$ has the finite intersection property, while for all $\eta \in A \backslash B_{A}$ the family $\left\{U_{\xi}: \xi \in \Lambda_{A} \cup\{\eta\}\right\}$ does not have the finite intersection property. We define

$$
\phi_{A}: A \backslash B_{A} \rightarrow \mathscr{P}_{\omega}^{*}\left(\Lambda_{A}\right)
$$

as follows. Let $\eta \in A \backslash B_{A}$; there is a nonempty finite subset $F_{\eta}$ of $\Lambda_{A}$ such that

$$
\bigcap_{\xi \in F_{\eta}} U_{\xi} \cap U_{\eta}=\varnothing ;
$$

we set $\phi_{A}(\eta)=F_{\eta}$ for $\eta \in A \backslash B_{A}$. We set

$$
\mathscr{P}_{A}=\left\{B_{A}\right\} \cup\left\{\phi_{A}^{-1}(\{F\}): F \in \mathscr{P}_{\omega}^{*}\left(\Lambda_{A}\right)\right\}
$$

and we note that

$$
\mathscr{P}_{A} \text { is a partition of } A \text {, with }\left|\mathscr{P}_{A}\right| \leqslant \kappa^{\kappa} \text {. }
$$

We now apply Lemma 1.1 , with $\rho=\left(\kappa^{\kappa}\right)^{+}$; since $\kappa$ is regular it follows that $\kappa \ll \rho$ and since $\alpha$ is regular and $\sqrt[\kappa]{\alpha}>2$, it follows that $\rho \leqslant \alpha$; thus, there is a family $\left\{A_{i}: i<\kappa\right\}$ such that $\left|A_{i}\right|=\alpha$ for $i<\kappa, A_{i^{\prime}} \subset A_{i}$ for $i<i^{\prime}<\kappa$, and $A_{i+1} \in \mathscr{P}_{A_{i}}$ for $i<\kappa$. Thus, since $\left|A_{i}\right|=\alpha$ for all $i<\kappa$, we have

$$
A_{i+1}=\phi_{A_{i}}^{-1}\left(\left\{F_{i}\right\}\right)
$$

for some $F_{i} \in \mathscr{P}_{\omega}^{*}\left(\Lambda_{A_{i}}\right), i<\kappa$. We set

$$
V_{i}=\bigcap_{\xi \in F_{i}} U_{\xi} \text { for } i<\kappa,
$$

and it is easy to verify that the family $\left\{V_{i}: i<\kappa\right\}$ consists of pairwise disjoint nonempty open subsets of $X$. This is a contradiction to the fact that $S(X)=\kappa$.

3.7. Lemma Let $\alpha, \kappa$ be infinite cardinals with $\kappa$ regular, $\operatorname{cf}(\alpha)<\kappa$, and $\alpha$ strongly $\kappa$-inaccessible. Then there is a family $\left\{\alpha_{\sigma}: \sigma<\operatorname{cf}(\alpha)\right\}$ of cardinals such that $\alpha_{\sigma}$ is regular, $\alpha_{\sigma}<\alpha, \alpha_{\sigma}$ is strongly $\kappa$-inaccessible for $\sigma<\operatorname{cf}(\alpha)$, and $\alpha=\Sigma_{\sigma<\operatorname{cf}(\alpha)} \alpha_{\sigma}$.

Proof. Let $\left\{\beta_{\sigma}: \sigma<\operatorname{cf}(\alpha)\right\}$ be a family of regular cardinals, $\beta_{\sigma}<\alpha$ and $\sigma=$ $\sum_{\sigma<\mathrm{cf}(\alpha)} \beta_{\sigma}$.

For every $\sigma<\operatorname{cf}(\alpha)$ we claim that $\beta_{\sigma}^{\kappa}<\alpha$.

In fact assuming that this is false, then there is $\sigma_{0}<\operatorname{cf}(\alpha)$ such that $\beta_{\sigma_{0}}^{\kappa} \geqslant \alpha$. But then we will have $\beta_{\sigma_{0}}^{\kappa}>\alpha$. (Really, if $\beta_{\sigma_{0}}^{\kappa}=\alpha$ since $\kappa$ is a regular cardinal we have that

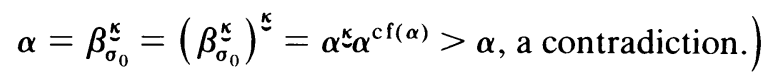

That contradicts the fact $\alpha$ is strongly $\kappa$-inaccessible and this proves the claim. 
Now setting $\alpha_{\sigma}=\left(\beta_{\sigma}^{\kappa}\right)^{+}$, we can easily verify that the family $\left\{\alpha_{\sigma}: \sigma<\operatorname{cf}(\alpha)\right\}$ satisfies the conclusion of the lemma.

Remark. Combining Corollary 1.3 and Lemma 3.7 we observe that if $\alpha$ is strongly $\kappa$-inaccessible cardinal and $\kappa$ is regular uncountable cardinal and $\operatorname{cf}(\alpha) \neq \kappa$, then always there is a family $\left\{\alpha_{\sigma}: \sigma<\operatorname{cf}(\alpha)\right\}$ of regular strongly $\kappa$-inaccessible cardinals with $\sum \alpha_{\sigma}=\alpha$. In the remaining case $\operatorname{cf}(\alpha)=\kappa$ the above family exists if $\kappa$ is a successor cardinal while in case $\kappa$ is a limit cardinal it does not exist.

3.8. Definition. Let $X$ be a topological space, and let $\mathcal{Q}=\left\{U_{\xi}: \xi \in \alpha\right\}$ be a family of nonempty open subsets of $X$. An index $\bar{\xi}<\alpha$ is called a representative of $\mathcal{Q}$ if the following condition is satisfied:

If $\beta<\alpha$ and if $\left\{B_{\xi}: \zeta<\beta\right\}$ is a family of nonempty open subsets of $U_{\xi}$, then there is $A \subset \alpha$, with $|A|=\alpha$, such that the family $\left\{U_{\xi} \cap B_{\xi}: \xi \in A\right\}$ has the finite intersection property for all $\zeta<\beta$.

Thus, Lemma 2.1 says that, if $S(X)=\kappa \ll \alpha$, and $\alpha$ is regular, then every family $\mathscr{Q}=\left\{U_{\xi}: \xi \in \alpha\right\}$ of nonempty open subsets of $X$ has a representative.

3.9. TheOREM. Let $X$ be a space $S(X)=\kappa \geqslant \omega$, and let $\alpha$ be a regular cardinal with $\kappa<\alpha$. Then $X$ has precaliber $(\alpha, \omega \cdot \sqrt[\kappa]{\alpha})$.

Proof. If $\sqrt[\kappa]{\alpha}=2$, then according to Rosenthal's result [5], $X$ has precaliber $(\alpha, \omega)$. If $\sqrt[\kappa]{\alpha}=\alpha$, then $\alpha$ is regular and $\alpha \gg \kappa$, and hence from Theorem 2.5, $X$ has precaliber $(\alpha, \alpha)$.

So we assume from now on that $2<\sqrt[\kappa]{\alpha}=\beta<\alpha$. Let $\left\{U_{\xi}: \xi<\alpha\right\}$ be a family of nonempty open subsets of $X$. We note that $\beta \gg \kappa$ and $\operatorname{cf}(\beta)<\kappa$, by Corollary 3.4 and Remark 3.5, and hence from Lemma 3.7, there is a family $\left\{\beta_{\sigma}: \sigma<\operatorname{cf}(\beta)\right\}$ of cardinals such that

$$
\beta_{\sigma} \text { is regular, } \quad \beta_{\sigma}<\beta, \quad \kappa \ll \beta_{\sigma} \text { for } \sigma<\operatorname{cf}(\beta),
$$

and

$$
\beta=\sum_{\sigma<\mathrm{cf}(\beta)} \beta_{\sigma} .
$$

Claim. There is a family $\left\{A_{\sigma}: \sigma<\operatorname{cf}(\beta)\right\}$ of subsets of $\alpha$ such that $\left|A_{\sigma}\right|=\beta_{\sigma}$ and the family $\left\{\mathcal{Q}_{\xi}: \xi \in A_{\sigma}\right\}$ has a representative $\xi_{\sigma}$ such that the family $\left\{U_{\xi_{\sigma}}: \sigma<\operatorname{cf}(\beta)\right\}$ has the finite intersection property.

Indeed, let $A$ be a subset of $\alpha$ with $|A|=\alpha$ and the property of Lemma 3.6. We proceed inductively. Let $\sigma<\operatorname{cf}(\beta)$. We assume that $A_{\tau}, \xi_{\tau}$ have been defined for $\tau<\sigma$ such that $A_{\tau} \subset A,\left|A_{\tau}\right|=\beta_{\tau},\left\{\mathcal{Q}_{\xi}: \xi \in A_{\tau}\right\}$ has the finite intersection property.

Let $\Lambda=\left\{\xi_{\tau}: \tau<\sigma\right\}$; thus $|\Lambda|<\operatorname{cf}(\beta) \leqslant \kappa^{\kappa}$; hence, from the characteristic property of $A$, as in Lemma 3.6, it follows that the set

$$
B=\left\{\eta \in A:\left\{U_{\xi}: \xi \in \Lambda \cup\{\eta\}\right\} \text { has the finite intersection property }\right\}
$$

has cardinality $\alpha$.

We choose $A_{\sigma} \subset B$ with $\left|A_{\sigma}\right|=\beta_{\sigma}$ and a $\xi_{\sigma}$ in $A_{\sigma}$ such that $\left\{\mathcal{Q}_{\xi}: \xi \in A_{\sigma}\right\}$ has representative $\xi_{\sigma}$. So the inductive definition is complete. 
Now setting $A_{1}=\cup_{\sigma<\mathrm{cf}(\alpha)} A_{\sigma}$ we claim that there is $A_{2} \subset A_{1}$ with $\left|A_{2}\right|=\beta$ such that the family $\left\{U_{\xi}: \xi \in A_{2}\right\}$ has the finite intersection property. Indeed, let $C$ be a maximal subset of $A_{1}$ such that $\left\{\xi_{\sigma}: \sigma<\operatorname{cf}(\beta)\right\} \subset C$ and $\left\{U_{\xi}: \xi \in C\right\}$ has the finite intersection property. It is enough to show that $|C| \geqslant \beta$. Suppose on the contrary that $|C|<\beta$. Then $|C|<\beta_{\sigma}$ for some $\sigma<\operatorname{cf}(\beta)$. Now the fact that $\xi_{\sigma} \in C$ is a representative of $\left\{U_{\xi}: \xi \in A_{\sigma}\right\}$ allows us to find $\eta \in A_{\sigma} \backslash C$ such that $\left\{U_{\xi}: \xi \in C \cup\right.$ $\{\eta\}\}$ has the finite intersection property, contradicting the maximality of $C$.

Incidentally we give a simple proof of Rosenthal's result which states that

3.10. Proposition. If $\alpha$ is a regular cardinal and $\alpha \geqslant S(X)$, then $X$ has $(\alpha, \omega)$ precaliber.

Proof. Let $\left\{U_{\xi}: \xi \in \alpha\right\}$ be a family of nonempty subsets of $X$. Inductively we choose sequences

$$
\left\{U_{\xi_{1}}, U_{\xi_{2}}, \ldots, U_{\xi_{n}}, \ldots, n<\omega\right\}, \quad\left\{A_{1}, A_{2}, \ldots, A_{n}, \ldots, n<\omega\right\}
$$

such that

(i) $\alpha \supset A_{1} \supset A_{2} \supset \cdots \supset A_{n} \supset \cdots n<\omega$ and $\left|A_{n}\right|=\alpha$,

(ii) $\cap{ }_{i=1}^{n} U_{\xi_{i}} \cap U_{\zeta} \neq \varnothing$ for all $\zeta \in A_{n}$.

This choice is possible from the regularity of $\alpha$ and the fact that $\alpha \geqslant \kappa=S(X)$.

Now the family $\left\{U_{\xi_{n}}: n<\omega\right\}$ obviously has the finite intersection property.

4.

4.1. THEOREM. Let $\beta$ be a strong limit cardinal with $\operatorname{cf}(\beta)=\omega$, and assume that $2^{\beta}=\beta^{+}$. Then there is an extremally disconnected compact Hausdorff space $\Omega$ such that $\Omega$ has a strictly positive measure (and hence, satisfies the c.c.c.) and $\Omega$ does not have caliber $\beta^{+}$.

Proof. Let $\lambda$ be the product measure that is inducted on the space $\{0,1\}^{\beta}$ by the coordinate measures $\left\{\lambda_{\xi}: \xi<\beta\right\}$, where $\lambda_{\xi}(\{0\})=\lambda_{\xi}(\{1\})=\frac{1}{2}$ for all $\xi<\beta$. Let also $\delta$ be the $\sigma$-algebra of $\lambda$-measurable subsets of $\{0,1\}^{\beta}$.

Claim 1. If $A$ is a subset of $\{0,1\}^{\beta}$ with $|A| \leqslant \beta$ then $A \in S$ and $\lambda(A)=0$. Indeed, since $\operatorname{cf}(\beta)=\omega$ it follows that $A=\cup_{n<\omega} A_{n}$ such that $\left|A_{n}\right|<\beta$ for all $n<\omega$, and hence it is enough to prove the claim for sets $A$ with $|A|<\beta$.

Let $A=\left\{x_{\sigma}: \sigma<\gamma\right\}, \gamma<\beta$, be a well ordering of $A$ and for $\zeta<\beta$ we set $\Lambda_{\zeta} \subset A$ such that $\Lambda_{\zeta}=\left\{x_{\sigma}: x_{\sigma}(\zeta)=0\right\}$. Since $\beta$ is strong limit it follows that $2^{|\gamma|}<\beta$ and thus there is $D_{A} \subset \beta$ such that $\left|D_{A}\right| \geqslant \omega$ and if $\zeta_{1}, \zeta_{2} \in D_{A}, \Lambda_{\zeta_{1}}=\Lambda_{\zeta_{2}}$. Let $B=$ $\left\{x_{1}, x_{2}\right\} \times\{0,1\}^{\beta \backslash D_{A}}$ where $x_{1}(\zeta)=0, x_{2}(\zeta)=1$ for all $\zeta \in D_{A}$. We easily verify that $A \subset B$ and from well-known facts on properties of product measure it follows that $\lambda(B)=0$.

We next claim that there is a family $\left\{K_{\eta}: \eta<\beta^{+}\right\}$of subsets of $\{0,1\}^{\beta}$ such that $K_{\eta}$ is compact for $\eta<\beta^{+}, \lambda\left(K_{\eta}\right)>0$ for $\eta<\beta^{+}$, and if $A \subset \beta^{+},|A|=\beta^{+}$then the family $\left\{K_{\eta}: \eta \in A\right\}$ does not have the finite intersection property.

Indeed, let $\left\{x_{\xi}: \xi<\beta^{+}\right\}$be a one-to-one enumeration of $\{0,1\}^{\beta}$, using the hypothesis $2^{\beta}=\beta^{+}$. We note that the set $\left\{x_{\xi}: \xi<\eta\right\}$ has cardinality $|\eta| \leqslant \beta$ for all 
$\eta<\beta^{+}$and hence from the statement proved above

$$
\lambda\left(\left\{x_{\xi}: \xi<\eta\right\}\right)=0 \text { for all } \eta<\beta^{+} .
$$

By the regularity of (the Haar measure) $\lambda$ for $\eta<\beta^{+}$there is a compact subset $K_{\eta} \subset\left\{x_{\xi}: \xi \geqslant \eta\right\}$ with $\lambda\left(K_{\eta}\right)>0$. Let $A \subset \beta^{+}$with $|A|=\beta^{+}$, then it is easy to verify that $\bigcap_{\eta \in A} K_{\eta}=\varnothing$.

Now for $\eta<\beta^{+}$let $\left[K_{\eta}\right]$ denote the $\Re$-equivalence class of $K_{\eta}$ and $U_{\eta}$ denote the unique open-and-closed subset of $\Omega$ that corresponds to $\left[K_{\eta}\right]$ via Stone's duality theorem.

Let $A \subset \beta^{+}$with $|A|=\beta^{+}$. We claim that $\left\{U_{\eta}: \eta \in A\right\}$ does not have the finite intersection property. This is equivalent to the claim that $\left\{\left[K_{\eta}\right]: \eta \in A\right\}$ does not have the finite intersection property. This follows from the fact that $\bigcap_{\eta \in A} K_{\eta}=\varnothing$. The proof is complete.

4.2. Corollary. Assume the Generalised Continuum Hypothesis, and let $\alpha$ be a cardinal such that $\operatorname{cf}(\alpha)=\beta^{+}$and $\operatorname{cf}(\beta)=\omega$.

Then there is an extremally disconnected compact Hausdorff space $\Omega$ with the c.c.c. such that

$\Omega$ does not have caliber $\alpha$, but has caliber $(\alpha, \beta)$.

4.3. Remark. (a) Corollary 4.2 shows that Theorem 3.9 is the best possible (at least assuming the G.C.H.).

(b) The above example extends a well-known example of Erdös on nonexistence of $\omega^{+}$caliber for all c.c.c. compact spaces. Namely Erdös has proved that under the G.C.H. the Stone space of the Boolean algebra of measurable subsets of $[0,1]$ with Lebesgue measure modulo null sets fails to have $\omega^{+}$-caliber.

(c) It follows from Theorem 4.1 that (at least assuming the G.C.H.) there are compact spaces $\Omega$ satisfying the c.c.c. and that fail to have caliber for arbitrary large regular cardinals.

(d) In [1] an extension of Theorem 4.1 that has the following form is given:

THEOREM. Assume the G.C.H. Then for every $\alpha, \kappa$ cardinals with $\operatorname{cf}(\alpha)<\kappa$ there is an extremally disconnected space $\Omega_{\alpha}$ such that $S\left(\Omega_{\alpha}\right) \leqslant \kappa$ and $\Omega_{\alpha}$ does not have $\alpha^{+}$ caliber.

4.4. Remark. Let $X$ be a compact Hausdorff space, $\mu$ a regular Borel measure on $X$, and $\alpha$ a cardinal such that $\omega^{+} \ll \alpha$ and $\omega^{+} \ll \operatorname{cf}(\alpha)$, and $\left\{S_{\xi}: \xi<\alpha\right\}$ a family of $\mu$-measurable subsets of $X$ with $\mu\left(S_{\xi}\right)>0$ for $\xi<\alpha$. Then there is $A \subset \alpha$ with $|A|=\alpha$ and $\bigcap_{\xi \in A} S_{\xi} \neq \varnothing$.

(Indeed, let $\Omega$ be the Stone space of the measure algebra $L=\mathfrak{x} / \mathfrak{\Re}$ of the measure $\mu$. Then $\Omega$ has a strictly positive measure, hence satisfies the c.c.c., and thus $\Omega$ has caliber $\alpha$ (Theorem 2.5). From the regularity of the measure $\mu$ there is a compact set $K_{\xi}$ such that $K_{\xi} \subset S_{\xi}$ and $\mu\left(K_{\xi}\right)>0$, for $\xi<\alpha$. The $\mu$-equivalence class $\left[K_{\xi}\right]$ corresponds by Stone's duality to a (unique) nonempty open-and-closed subset of $\Omega$. Hence there is $A \subset \alpha$ with $|A|=\alpha$ such that $\left\{\left[K_{\xi}\right]: \xi \in A\right\}$ has the finite 
intersection property and since each $K_{\zeta}$ is a compact subset of $X$ it follows that $\cap_{\xi \in A} S_{\xi} \supset \cap_{\xi \in A} K_{\xi} \neq \varnothing$ as required.)

4.5. RemarK. Theorem 2.5 implies a negative, in general, answer to the Comfort problem on the determination of possible calibers for compact spaces. Indeed, let $H=\left\{\alpha_{\gamma}: \gamma \in \Gamma\right\}$ be a family of regular cardinals, $\omega \in H$ and if we want to find a compact space $X_{H}$ that does not have caliber exactly on the cardinals $\alpha$ such that $\operatorname{cf}(\alpha) \in H$, then the family $H$ need satisfy the following condition. If $\kappa$ is the least regular cardinal that does not belong to $H$, then obviously $S(X) \leqslant \kappa$ and therefore from Theorem 2.5 it follows that every regular $\alpha$ such that $\alpha \gg \kappa$ also does not belong to $H$.

However we do not know if for a family $H$ that satisfies the above condition always there is the space $X_{H}$. In particular we do not know even under the G.C.H. if for a given family $H$ of regular cardinals such that $\omega^{+} \notin H$ and for $\alpha \in H, \alpha$ has the form $\alpha=\beta^{+}, \operatorname{cf}(\beta)=\omega$, there is the compact space $X_{H}$.

5. This section is due to the referee and contains a coherent picture of what has been proved and what cases are left open.

5.1. Definition. For infinite cardinals $\alpha, \beta$, $\kappa$ let the symbol $\alpha \Rightarrow(\kappa, \beta)$ denote the statement: every space $X$ with $S(X) \leqslant \kappa$ has precaliber $(\alpha, \beta)$.

Let $\mathfrak{f}(\kappa, \beta)=\min \{\alpha: \alpha \Rightarrow(\kappa, \beta)\}$.

5.2. About function $\mathfrak{f}$. Some properties of the function $\mathfrak{f}$ are the following:

(a) $f(\kappa, \beta)$ is monotonically increasing in both arguments.

(b) $\mathfrak{f}(\omega, \beta)=\beta$ for every cardinal $\beta$.

(c) $f(\omega, \beta)=\beta$ for every $\beta$ (Proposition 3.10).

(d) If $\kappa$ is singular then $\mathfrak{f}(\kappa, \beta)=\sup \{\mathfrak{f}(\lambda, \beta): \lambda<\kappa\}$ (Erdös-Tarski theorem).

From now on, we assume for simplicity the G.C.H.; by Theorem 3.9 if $\gamma=$ $\max \{\kappa, \beta\}$ then $\gamma \leqslant f(\kappa, \beta) \leqslant \gamma^{+}$. We deal with the problem of deciding between $\gamma$ and $\gamma^{+}$.

Case 1. $\beta<\kappa$. Then $\mathrm{f}(\kappa, \beta)=\kappa$ if $\beta=\omega$ or if $\kappa$ is singular. If $\omega<\beta<\kappa=\operatorname{cf}(\kappa)$, we do not know anything about the values of $f(\kappa, \beta)$.

Case 2. $\beta=\kappa . f(\kappa, \kappa)=\kappa$ if $\kappa$ is a weakly compact cardinal (for example if $\kappa=\omega$ ) while $\mathfrak{f}(\kappa, \kappa)=\kappa^{+}$if $\kappa$ is a singular cardinal or a successor cardinal or if there is a $\kappa$ Souslin tree. Thus (by Jensen's result) assuming $V=L$, we have $f(\kappa, \kappa)=\kappa$ if and only if $\kappa$ is weakly compact. We do not know if this is true assuming only G.C.H.

Case 3. $\beta>\kappa$. If $\kappa=\omega$ then $\mathfrak{f}(\kappa, \beta)=\beta$. If $\operatorname{cf}(\beta)<\kappa$ then $\mathfrak{f}(\kappa, \beta)=\beta^{+}$. If $\operatorname{cf}(\beta)=\kappa$ then $\mathfrak{f}(\kappa, \beta)=\beta$ if and only if $\mathfrak{f}(\kappa, \kappa)=\kappa$ (Case 2). If $\operatorname{cf}(\beta)>\kappa$ and if $\operatorname{cf}(\beta)$ is a limit cardinal or else $\operatorname{cf}(\beta)=\gamma^{+}$with $\operatorname{cf}(\gamma) \geqslant \kappa$ then $\mathrm{f}(\kappa, \beta)=\beta$. If $\operatorname{cf}(\beta)=\gamma^{+}$and $\omega \leqslant \operatorname{cf}(\gamma)<\kappa$ then by Theorem 4.1 and [1] (see Remark 4.3(d)) it follows that $\mathfrak{f}(\kappa, \beta)=\beta^{+}$.

Finally, the following statements are easily seen to be equivalent for all cardinals $\alpha, \beta$ and $\kappa$.

(1) Every extremally disconnected compact Hausdorff space $X$ with $S(X) \leqslant \kappa$ has caliber $(\alpha, \beta)$.

(2) Every space $X$ with $S(X) \leqslant \kappa$ has precaliber $(\alpha, \beta)$. 
(3) Given any family $\left\{U_{\xi}: \xi \in \alpha\right\}$ of nonempty sets, either there is $B \subset \alpha$ such that $|B|=\beta$ and $\left\{U_{\xi}: \xi \in B\right\}$ has the finite intersection property, or else there is a family $\left\{V_{\eta}: \eta<\kappa\right\}$ of pairwise disjoint nonempty sets such that each $V_{\eta}$ is the intersection of finitely many $U_{\xi}$ 's.

(4) For every family $\mathscr{L} \subset \mathcal{P}(\alpha)$ with $2 \leqslant|X|<\omega$ for each $X \in \mathcal{L}$, either there is an independent set $B \subset \alpha$ with $|B|=\beta$, or else there is a family $\left\{A_{\xi}: \xi<\kappa\right\}$ of finite independent subsets of $\alpha$ such that $A_{\xi} \cup A_{\eta}$ is dependent for $\xi<\eta<\kappa$. (A set $A \subset \alpha$ is "independent" if $\varrho \cap \mathcal{P}(A)=\varnothing$; "dependent" if $\mathcal{L} \cap \mathscr{P}(A) \neq \varnothing$.)

Note that if we weaken (4) by requiring $|X|=2$ for $X \in \mathcal{L}$, it becomes a weakened version of the ordinary partition relation $\alpha \rightarrow(\kappa, \beta)^{2}$ of Erdös and Rado.

ACKNOWLedgment. We would like to express our thanks to Professor S. Negrepontis for his great help during the preparation of the paper. We are also grateful to the referee for his valuable suggestions on the material in it. His numerous comments helped us to improve parts of this paper; among others, following his suggestions, we simplified the proofs of Lemmas 1.1 and 2.4.

ADDED IN PROOF. Corresponding results for independent families of sets and their applications to Topology and Functional Analysis, proved by the authors, are contained in the paper, "Embeddings of $l^{1}(\Gamma)$ into subspaces of $C(\Omega)$ ", to appear in the Mathematical Proceedings of the Cambridge Philosophical Society. Similar results have been established by J. Shelah in Remarks on Boolean algebras, Algebra Universalis 11 (1980), 77-89.

\section{REFERENCES}

1. S. Argyros, Boolean algebras without free families, Algebra Universalis (to appear).

2. S. Broverman, J. Ginsburg, K. Kunen and F. D. Tall, Topologies determined by $\sigma$-ideals on $\omega_{1}$, Canad. J. Math. 6 (1978), 1306-1312.

3. W. W. Comfort, A survey of cardinal invariants, General Topology Appl. 1 (1971), 163-199.

4. J. Gerlits, manuscript notes, 1978.

5. H. P. Rosenthal, On injective Banach spaces and the spaces $L^{\infty}(\mu)$ for finite measures $\mu$, Acta Math. 124 (1970), 205-248.

6. N. A. Šanin, On the product of topological spaces, Trudy Mat. Inst. Steklov. 24 (1948), 1-112.

7. S. Shelah, Remarks on cardinal invariants in topologi, General topology Appl. 7 (1977), 251-259.

Chair I of Mathematical Analysis, University of Athens, Athens, Greece (621) 